\title{
Imaging of HIF-1-Active Tumor Hypoxia Using a Protein Effectively Delivered to and Specifically Stabilized in HIF-1-Active Tumor Cells
}

Takashi Kudo ${ }^{1}$, Masashi Ueda ${ }^{1,2}$, Yuji Kuge ${ }^{1}$, Takahiro Mukai ${ }^{3}$, Shotaro Tanaka ${ }^{4}$, Maki Masutani ${ }^{1}$, Yasushi Kiyono ${ }^{2,5}$, Shinae Kizaka-Kondoh ${ }^{4}$, Masahiro Hiraoka ${ }^{4}$, and Hideo Saji ${ }^{1}$

${ }^{I}$ Department of Patho-Functional Bioanalysis, Graduate School of Pharmaceutical Sciences, Kyoto University, Kyoto, Japan; ${ }^{2}$ Radioisotopes Research Laboratory, Faculty of Medicine, Kyoto University Hospital, Kyoto University, Kyoto, Japan; ${ }^{3}$ Department of Biomolecular Recognition Chemistry, Graduate School of Pharmaceutical Sciences, Kyushu University, Fukuoka, Japan; ${ }^{4}$ Department of Radiation Oncology and Image-Applied Therapy, Kyoto University Graduate School of Medicine, Kyoto University, Kyoto, Japan; and ${ }^{5}$ Biomedical Imaging Research Center, University of Fukui, Fukui, Japan

Hypoxia-inducible factor-1 (HIF-1) plays an important role in malignant tumor progression and in the development of resistance to radiotherapy. We designed a novel fusion protein (PTDODD-SAV [POS]) consisting of a protein transduction domain (PTD), streptavidin (SAV), and a portion of the oxygen-dependent degradation domain (ODD) of HIF-1 $\alpha$ that confers the same oxygen-dependent regulation as HIF-1 $\alpha$ on POS. (3-123/125Iiodobenzoyl)norbiotinamide (123/125I-IBB) was conjugated to the SAV moiety of POS to synthesize 123/125|-IBB-labeled POS (123/125I-IPOS). The purpose of this study was to evaluate the feasibility of ${ }^{123}$-IPOS as an imaging probe for HIF-1-active tumor hypoxia. Methods: After a 24-h incubation of ${ }^{125}$ I-IPOS with various tumor cell lines under either normoxic $\left(20 \% \mathrm{O}_{2}\right)$ or hypoxic $\left(0.1 \% \mathrm{O}_{2}\right)$ conditions, the intracellular radioactivity was investigated. Then, the biodistribution of ${ }^{123 / 125}$ I-IPOS was examined with tumor-implanted mice, and an in vivo imaging study was performed. The tumoral accumulation of ${ }^{125}$ I-IPOS was compared with HIF-1 activity using the mice carrying tumors with the HIF-1-dependent luciferase reporter gene. Furthermore, the intratumoral localization of ${ }^{125}$ I-IPOS was examined by the autoradiographic study, and then the same slide was subjected to immunostaining for pimonidazole, which is the hypoxic marker. Results: The ratios of radioactivity in hypoxic cells to that in normoxic cells were more than 2 . These results indicate incorporation of ${ }^{125}$ I-IPOS into these cells and degradation of ${ }^{125}$ I-IPOS by normoxic tumor cells. In the biodistribution study, ${ }^{125}$ I-IPOS accumulated in the tumor $(1.4 \pm 0.3$ percentage injected dose per gram) $24 \mathrm{~h}$ after administration. At that time, 125I-IPOS showed high tumor-to-blood and tumor-to-muscle ratios (5.1 \pm 0.3 and $14.0 \pm 3.9$, respectively). The tumors were clearly visualized by in vivo imaging $24 \mathrm{~h}$ after ${ }^{123}$ I-IPOS injection (tumor-to-muscle ratio was 9.6). The tumoral accumulation of 125I-IPOS correlated with HIF-1 activity $(R=0.71, P<0.05)$,

Received Dec. 14, 2008; revision accepted Feb. 16, 2009.

For correspondence or reprints contact: Hideo Saji, Department of PathoFunctional Bioanalysis, Graduate School of Pharmaceutical Sciences, Kyoto University, 46-29 Yoshida-Shimo-Adachi-cho, Sakyo-ku, Kyoto 606-8501, Japan.

E-mail: hsaji@pharm.kyoto-u.ac.jp

COPYRIGHT () 2009 by the Society of Nuclear Medicine, Inc. and its intratumoral distribution coincided with the hypoxic regions. Conclusion: ${ }^{123}$ I-IPOS is a potential probe for the imaging of HIF-1 activity in tumors. Given the role of HIF-1 in tumor biology, its detection may be considered an indicator of aggressive cancer phenotypes.

Key Words: molecular imaging; oncology; radiopharmaceuticals; hypoxia-inducible factor-1 (HIF-1); tumor hypoxia; oxygendependent degradation (ODD); protein transduction domain (PTD)

J Nucl Med 2009; 50:942-949

DOI: 10.2967/jnumed.108.061119

$\mathbf{I}_{\mathrm{n}}$ n solid tumors, hypoxia, or an oxygen tension below physiologic levels, develops as abnormal proliferation outstrips the blood supply (1). This hypoxic region is involved in tumor malignancy and proliferation and results in the development of resistance to radiotherapy (2-4). Hypoxia-inducible factor-1 (HIF-1), a transcription factor that regulates the cellular response to hypoxia, induces several genes that mediate these changes in tumor behavior $(5,6)$. Indeed, HIF1 -active or hypoxic cells have recently been shown to play crucial roles in angiogenesis and radioresistance (7). Finally, the expression level of HIF-1 correlates with a poor prognosis in many tumors $(8,9)$. Thus, imaging HIF-1-active regions in a tumor has the potential to provide, in a noninvasive manner, insight into those aspects of tumor biology most relevant to treatment design.

HIF-1 is a heterodimer that consists of the oxygen-sensitive $\alpha$-subunit (HIF-1 $\alpha$ ) and the constitutively expressed $\beta$-subunit (HIF-1 $\beta$ ). Under normoxic conditions, HIF- $1 \alpha$ is hydroxylated by prolyl hydroxylases on the proline residues in the oxygen-dependent degradation domain (ODD). The hydroxylated proline residues accelerate ubiquitination of ODD 
and subsequent degradation of HIF-1 $\alpha$ (10). HIF- $1 \alpha$ expression is regulated in an oxygen-dependent manner at the posttranscriptional level and is responsible for regulation of HIF-1 activity (11).

We recently developed TOP3 (TAT-ODD-procaspase-3), a fusion protein with 3 domains: TAT-PTD, ODD, and procaspase-3 (12-16). The protein transduction domain (PTD) is derived from the PTD of the HIV type 1 TAT protein (17) and efficiently delivers TOP 3 to any tissue in vivo. The ODD domain contains a von Hippel Lindau-mediated protein destruction motif of human HIF-1 $\alpha$ protein (18) and confers hypoxia-dependent stabilization on TOP3 (14). Intraperitoneal injection of TOP3 into tumor-bearing mice results in specific targeting of HIF-1-active tumor cells $(7,12)$. These results demonstrated that PTD-ODD fusion proteins were efficiently delivered to hypoxic regions and stabilized in HIF1 -active cells.

In this study, we took advantage of the PTD-ODD fusion proteins for imaging of HIF-1-active hypoxic tumors. To label PTD-ODD with a radioactive reagent, PTD3-ODD was fused to a monomeric streptavidin (SAV). The SAV component was then used to join the compound to a radiolabeled biotin derivative, (3-123 I-iodobenzoyl)norbiotinamide ( ${ }^{123} \mathrm{I}-$ IBB). Our concept of hypoxia imaging is summarized in Figure 1. In this study, we evaluated the feasibility of ${ }^{123} \mathrm{I}-$ IBB-labeled PTD-ODD-SAV (123I-IPOS) as an imaging probe for HIF-1-active tumor hypoxia.

\section{MATERIALS AND METHODS}

\section{Construction of Plasmid and Preparation of Recombinant Protein POS}

To construct the pGEX/POS plasmid encoding the POS fusion protein, the cDNAs encoding PTD3 and $\mathrm{ODD}_{548-603}$ were constructed as described previously $(18,19)$. The cDNA encoding the

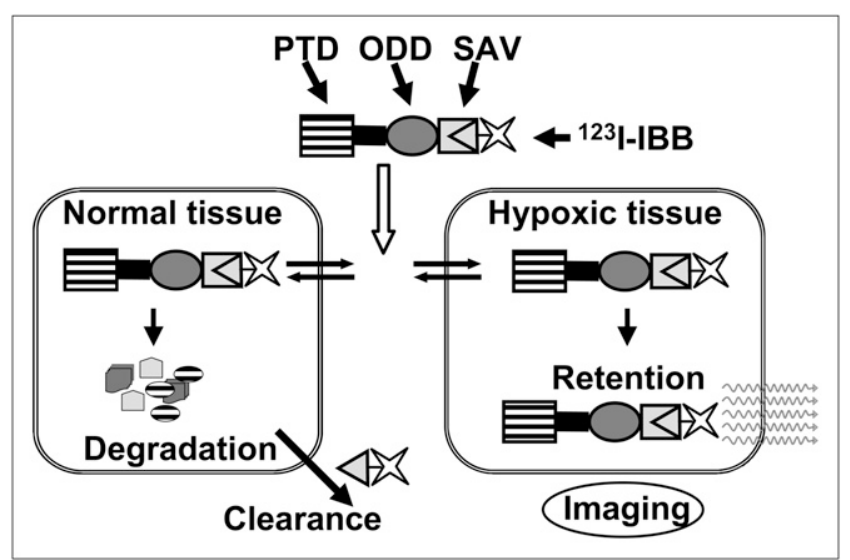

FIGURE 1. Concept of hypoxia imaging using 123/-IPOS. PTD enables ${ }^{123}$ I-IPOS to be delivered to normoxic and hypoxic tissue. In normoxic tissue, POS is degraded in manner similar to HIF-1 $\alpha$ degradation, and ${ }^{123}$-IBB is cleared. In contrast, in HIF-1-active tissues, POS escapes degradation and radioactivity is retained within cell. Thus, POS enables specific imaging of HIF-1-active hypoxic region. spacer sequence VDVADLEHD was constructed by annealing synthesized oligonucleotides corresponding to the polypeptide to BglII and BamHI endonuclease restriction sites at the 5' and $3^{\prime}$ sites, respectively. SAV cDNA was amplified by polymerase chain reaction using corresponding oligonucleotides as primers and a bacterial genome as a template. The resultant cDNA encoded the SAV protein from its 25th amino acid to the $\mathrm{C}$ terminus and had $\mathrm{KpnI}$ and $\mathrm{XhoI}$ endonuclease restriction sites at the $5^{\prime}$ and $3^{\prime}$ sites, respectively. The cDNA encoding POS was constructed by ligating the cDNAs constructed above into the EcoRI and XhoI sites of the pGEX6P-3 vector (GE Healthcare Bioscience). The resultant pGEX/POS vector encoded GST-tagged POS and was transformed into BL21(DE3)plysS (Novagen). GST-POS recombinant protein was induced with $1 \mathrm{mM}$ isopropyl- $\beta$-d-thiogalactopyranoside for $24 \mathrm{~h}$ at $4^{\circ} \mathrm{C}$ and purified over a glutathatione Sepharose $4 \mathrm{~B}$ column (GE Healthcare Bioscience). The GST-tag was removed from the recombinant protein with PreScission Protease (GE Healthcare Bioscience) in the column. The eluate containing POS was then dialyzed against $10 \mathrm{mM}$ Tris-hydrochloric acid buffer ( $\mathrm{pH}$ 8.0) and concentrated using the Amicon Ultra-15 5k NMWL device (Millipore). The purity and molecular weight of POS were determined by sodium dodecylsulfate-polyacrylamide gel electrophoresis and Coomassie staining.

\section{Synthesis of IBB}

IBB was prepared as previously described (20). The products were obtained as white crystals and stored under argon at $4^{\circ} \mathrm{C}$ (yield, 30.5\%).

\section{Radiosynthesis of ${ }^{123}$ |-||BB and ${ }^{125|-| \mid B B}$}

Ammonium ${ }^{123}$ I-iodide was kindly provided by Nihon MediPhysics. Sodium ${ }^{125}$ I-iodide was purchased from Perkin Elmer Life and Analytic Sciences. All other chemicals used were of reagent grade. A total of $1 \mathrm{GBq} \mathrm{NH}_{4}{ }^{123}$ I or $37 \mathrm{MBq} \mathrm{Na}^{125}$ I were added to a vial containing $N$-succinimidyl 3-(tributhylstannyl)benzoate (72.2 $\mu \mathrm{L}, 2 \mathrm{mg} / \mathrm{mL}$ of $1 \%$ acetic acid methanol) and $N$-chlorosuccinimide ( $19.8 \mu \mathrm{L}, 0.5 \mathrm{mg} / \mathrm{mL}$ of methanol). The vial was vortexed and the reaction allowed to proceed at room temperature for $30 \mathrm{~min}$. $\mathrm{NaHSO}_{3}\left(3.2 \mu \mathrm{L}, 0.72 \mathrm{mg} / \mathrm{mL}\right.$ in $\left.\mathrm{H}_{2} \mathrm{O}\right)$ was added to the product, which was vortexed before being concentrated under a stream of nitrogen. A solution of norbioinamine-hydrochloric acid in dimethylformamide:water $(4: 1)(100 \mu \mathrm{L}, 3 \mathrm{mg} / \mathrm{mL})$ was added to the vial. The reaction was incubated at room temperature for $2 \mathrm{~h}$, and the sample was purified with high-performance liquid chromatography (HPLC) (Cosmosil 5C 18 -AR-300 column, $4.6 \mathrm{~mm} \times 15 \mathrm{~cm}$; Nacalai Tesque) ( $25 \%$ acetonitrile, $1 \mathrm{~mL} / \mathrm{min}$; wave length, $254 \mathrm{~nm})$. The retention time of IBB was $21 \mathrm{~min}$. The solvent was evaporated and was reconstituted in water for use in the in vitro and in vivo studies. The radiochemical purity was confirmed with HPLC using the same method as was used for purification.

\section{Cell Culture}

MM1, HeLa, and Suit2 cells were obtained from American Type Culture Collection. FM3A cells were purchased from the Health Science Research Resources Bank, and MDA-MB-231 cells were purchased from Dainippon Sumitomo Pharma Co., Ltd. MM1, HeLa, and Suit2/HRE-luciferase (19) cells were maintained in 10\% fetal bovine serum-Dulbecco's modified Eagle's medium (Nissui Pharmaceutical). FM3A and MDA-MB-231 cells were cultured in $10 \%$ fetal bovine serum-RPMI medium (Nissui Pharmaceutical). The culture media were supplemented with penicillin (100 units/ $\mathrm{mL})$ and streptomycin $(100 \mu \mathrm{g} / \mathrm{mL})$. Cells were incubated at $37^{\circ} \mathrm{C}$ in 
a well-humidified incubator with $5 \% \mathrm{CO}_{2}$ and $95 \%$ air for normoxic culture or incubated in an anaerobic chamber (Concept Mini MACS; Biotrace Limited) for hypoxic culture.

\section{Binding of ${ }^{125}$-IBB to POS}

To prepare ${ }^{125} \mathrm{I}-\mathrm{IPOS},{ }^{125} \mathrm{I}-\mathrm{IBB}$ was added to a microtube containing POS (100 $\mu \mathrm{L}, 13.6 \mathrm{ng} / \mathrm{mL})$. After a 1-h incubation, the reactant was purified by size-exclusion HPLC (TSKgel SuperSW2000 $[4.6 \mathrm{~mm} \times 30 \mathrm{~cm}]$ connected by the TSKguardcolumn SuperSW [4.6 mm $\times 3.5 \mathrm{~cm}$ ]; Tosoh Corp.) $(100 \mathrm{mM}$ phosphate buffer [pH 6.8], $0.2 \mathrm{~mL} / \mathrm{min}$; wave length, $254 \mathrm{~nm}$ ). The radiochemical purity was examined by paper chromatography developed with saline $\left(R_{\mathrm{f}}=0\right)$.

\section{Biotin Competitive Assay}

${ }^{125} \mathrm{I}-\mathrm{IBB}, \mathrm{D}-$ biotin $(0-40 \mathrm{pmol})$, and $100 \mathrm{mM}$ citrate buffer $(\mathrm{pH}$ 5.0) were added to a microtube containing POS (1.36 ng). The reaction was performed at $37^{\circ} \mathrm{C}$ for $1 \mathrm{~h}$. The reaction mixture was applied to an Amicon Microcon filter (Millipore) and centrifuged at $4^{\circ} \mathrm{C}$ and $4,500 \mathrm{~g}$ for $30 \mathrm{~min}$ (Micro Cooling Centrifuge 1720; KUBOTA). The radioactivities of the reactant and filtrate were then measured and the binding rate calculated.

\section{Cellular Assay}

HeLa, MM1, and FM3A cells were transfected with the pGL3/

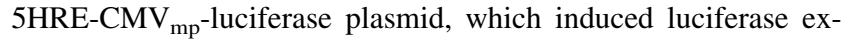
pression in response to HIF-1 activity (12). Transfection was performed using reagent (Lipofectamin 2000; Invitrogen). After a 24-h incubation under normoxic $\left(20 \% \mathrm{O}_{2}\right)$ or hypoxic $\left(0.1 \% \mathrm{O}_{2}\right)$ conditions, cells were washed and lysed with Reporter Lysis Buffer (Promega), and luciferase activity was evaluated with a single tube luminometer (Lumat LB 9507; Berthold) using a Luciferase Assay System (Promega). The protein concentration was measured with the BCA Protein Assay Kit (Pierce) for normalization.

ODD-dependent, normoxic, POS degradation was evaluated by 2 assays. In the first assay, MM1, HeLa, and FM3A cells were incubated in advance for more than $6 \mathrm{~h}$ under either normoxic or hypoxic conditions, after which ${ }^{125}$ I-IPOS $(0.28 \mu \mathrm{g}, 37 \mathrm{kBq})$ was added. Cells were then incubated for an additional $24 \mathrm{~h}$ in their respective culture conditions. After the cells were washed and lysed, the radioactivity of the lysate was measured using an auto well $\gamma$-counter (ARC2000; Aloka), and the protein concentration was measured and used for normalization. In the second assay, HeLa cells were incubated in advance for more than $6 \mathrm{~h}$ under hypoxic conditions, after which ${ }^{125}$ I-IPOS $(0.28 \mu \mathrm{g}, 37 \mathrm{kBq})$ was added. The cells were then incubated for an additional $24 \mathrm{~h}$ under hypoxic conditions. After washing, the medium was replaced with fresh medium, and the cells were subjected to further incubation for 3 or $24 \mathrm{~h}$ under normoxic or hypoxic conditions. After the incubation, cells were washed and lysed, and the radioactivity and protein concentration of the lysate were measured. The radioactivities of the cells and medium were analyzed by ultrafiltration (Amicon Microcon filter; Millipore) and size-exclusion HPLC. All measurements were performed in triplicate.

\section{Animal Model}

Animal studies were conducted in accordance with our institutional guidelines, and the experimental procedures were approved by the Kyoto University Animal Care Committee. To produce the mouse models of tumor implantation, $5 \times 10^{6} \mathrm{FM} 3 \mathrm{~A}$ cells in $100 \mu \mathrm{L}$ of phosphate-buffered saline (PBS) were subcutaneously implanted into the right thighs of 5-wk-old female $\mathrm{C} 3 \mathrm{H} / \mathrm{He}$ mice (Japan SLC,
Inc.). A total of $1 \times 10^{7}$ MDA-MB-231 cells or $5 \times 10^{6}$ Suit $2 / \mathrm{HRE}-$ luciferase cells in $100 \mu \mathrm{L}$ of PBS were subcutaneously implanted into the right thighs of 5-wk-old female BALB/c nu/nu mice (Japan SLC, Inc.). After the cells implanted, the mice were maintained on the AIN76-A-based biotin-free diet (Japan SLC, Inc.). FM3A and Suit 2 tumor models were used 2 wk after implantation, and the MDA-MB-231 tumor models were used 1 mo after implantation for the tracer study.

\section{Biodistribution}

${ }^{125}$ I-IPOS (0.05-30 $\mu \mathrm{g}, 37 \mathrm{kBq} /$ mouse) or ${ }^{125} \mathrm{I}$-IBB $(37 \mathrm{kBq} /$ mouse) was injected intravenously into FM3A-implanted mice. At the appropriate time points after administration, the mice were sacrificed. Whole-organ specimens were immediately removed and weighed, and the radioactivity was measured. The results were expressed as the percentage injected dose per gram $(\% \mathrm{ID} / \mathrm{g})$.

\section{In Vivo Imaging}

${ }^{123} \mathrm{I}-\mathrm{IPOS}(30 \mu \mathrm{g}, 5.4-16 \mathrm{MBq})$ was injected in FM3Aimplanted mice via the tail vein. The mice were anesthetized with $2.5 \%$ halothane and placed on the scanner bed in the prone position. Planar images were obtained for $10 \mathrm{~min}$ at 24 and $48 \mathrm{~h}$ after injection as previously described (21). Regions of interest were set on the tumor in the right thigh and the corresponding area in the left thigh.

\section{Tumoral Accumulation of ${ }^{125}$ I-IPOS Versus HIF-1 Transcriptional Activity in Vivo}

The Suit2/HRE-luciferase-implanted mice were intravenously injected with ${ }^{125} \mathrm{I}$-IPOS ( $30 \mu \mathrm{g}, 37 \mathrm{kBq} /$ mouse). After $24 \mathrm{~h}, 200 \mu \mathrm{L}$ of D-luciferin solution ( $10 \mathrm{mg} / \mathrm{mL}$ in PBS; Promega) was injected intraperitoneally. After $20 \mathrm{~min}$, the mice were anesthetized with $2.5 \%$ isoflurane and imaged using the IVIS Spectrum System (Xenogen) to measure the luciferase activity as the externally detected photon count. The photon counts within the tumors were analyzed with software (Living Image 3.0; Xenogen). After luciferase imaging, the mice were sacrificed and the tumors immediately removed. The radioactivity was then measured and the results expressed as the percentage injected dose (\%ID).

\section{Autoradiography}

Autoradiographic studies of ${ }^{125}$ I-IPOS were performed in MDAMB-231-implanted mice. ${ }^{125} \mathrm{I}-\mathrm{IPOS}$ (30 $\mu \mathrm{g}, 2.5 \mathrm{MBq} / \mathrm{mouse}$ ) was injected intravenously, and at $22 \mathrm{~h}$ after injection pimonidazole $(60 \mathrm{mg} / \mathrm{kg})$ was injected intraperitoneally. Mice were sacrificed $2 \mathrm{~h}$ later. Autoradiograms were obtained as previously described (21), with the following slight modification: BAS5000 instead of BAS3000.

\section{Immunohistochemistry}

The slides used in the autoradiographic study were subjected to immunostaining for pimonidazole. The immunostaining was performed using the Hypoxyprobe-1 Plus Kit (Chemicon), according to the manufacturer's protocol. To evaluate the specificity of the signal, tumor sections derived from pimonidazole-untreated mice were also stained by following the same protocol.

\section{Statistical Analyses}

Comparisons between 2 groups were performed with the MannWhitney $U$ test. Correlation coefficients were assessed using the Spearman rank analysis. A $P$ value of less than 0.05 was considered statistically significant. 


\section{RESULTS}

Construction of the Fusion Protein POS

The recombinant protein POS consisted of 3 parts: PTD, ODD, and SAV (Fig. 1). The PTD was composed of polylysine and hydrophobic amino acids, and was named PTD3. The ODD was $\mathrm{ODD}_{548-603}$, the same as the one previously described in TOP3 (7). The spacer sequence VDVADLEHD was inserted between PTD and ODD for stabilization of the fusion protein. The molecular weight of the fusion protein was approximately $34 \mathrm{kDa}$ (Fig. 2).

\section{Radiosynthesis of ${ }^{125}$ |-IBB and ${ }^{123}$ |-IBB}

${ }^{125} \mathrm{I}-\mathrm{IBB}$ and ${ }^{123} \mathrm{I}-\mathrm{IBB}$ were obtained in the absence of a carrier and with radiochemical yields of $65 \%$ and $29 \%$ and radiochemical purities of greater than $94 \%$ and greater than 95\%, respectively (Supplemental Fig. 1; supplemental materials are available online only at http://jnm.snmjournals.org).

\section{Binding of ${ }^{125}$ I-IBB to POS}

After the incubation of ${ }^{125} \mathrm{I}-\mathrm{IBB}$ with POS, the radioactivity was detected at about $34 \mathrm{kDa}$ by size-exclusion HPLC analysis, indicating that ${ }^{125} \mathrm{I}-\mathrm{IBB}$ bound to POS to form ${ }^{125} \mathrm{I}-$ IPOS (Fig. 3A). This binding was inhibited by D-biotin in a dose-dependent manner (Fig. 3B).

\section{Oxygen-Dependent Regulation of ${ }^{125}$ |-IPOS In Vitro}

To validate the stabilization of HIF- $1 \alpha$ in our experimental conditions, we performed a luciferase assay using an HREresponsive reporter system (12). The luciferase activities in HeLa, MM1, and FM3A cells, incubated under hypoxic conditions, were 28-, 17- and 29-fold higher than those of cells incubated under normoxic conditions, respectively (Fig. 4A).

We then added ${ }^{125}$ I-IPOS to the culture medium of HeLa, MM1, and FM3A cells and compared the radioactivity retained in the cells after incubation under normoxic conditions with that after incubation under hypoxic conditions for $24 \mathrm{~h}$. The ratios of the radioactivity in hypoxic cells to that in normoxic cells were 2.7, 2.2, and 2.3 in HeLa, MM1, and FM3A cells, respectively (Fig. 4B). Furthermore, the accumulated radioactivity under hypoxic conditions was de-

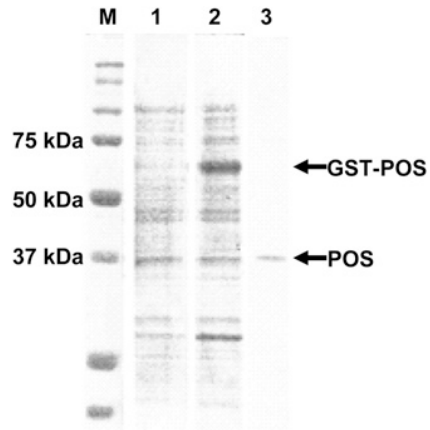

FIGURE 2. Analysis of fusion protein expression with sodium dodecylsulfate-polyacrylamide gel electrophoresis (lane M, molecular weight marker; lane 1, Escherichia coli lysate before IPTG induction; lane 2, E. coli lysate after IPTG induction; lane 3, protein solution after purification). New band was expressed in lane 2 at

about $60 \mathrm{kDa}$, corresponding to GST-fused POS. After purification, only 1 band was detected in lane 3 at $34 \mathrm{kDa}$. IPTG $=$ isopropyl- $\beta$-d-thiogalactopyranoside.
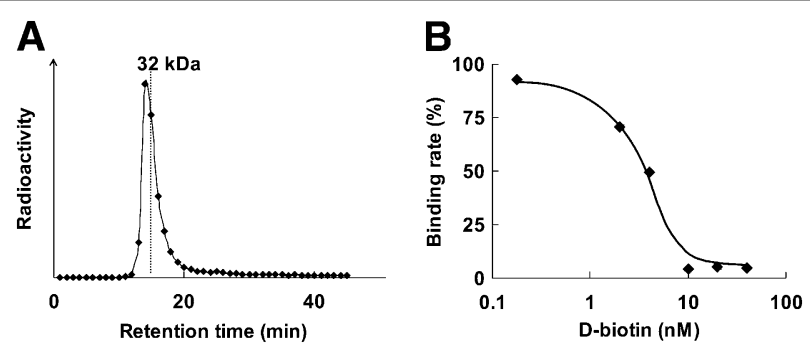

FIGURE 3. Binding of ${ }^{125} \mathrm{I}-\mathrm{IBB}$ to POS. (A) Size-exclusion HPLC chromatogram after incubation of ${ }^{125}$ I-IBB with POS. Symbols represent radioactivity of each eluate collected every $1 \mathrm{~min}$. (B) Concentration-dependent inhibition of ${ }^{125}$ I-IBB binding to POS by D-biotin. Symbols and bars represent mean and SD.

creased in a time-dependent manner after reoxygenation. After 24-h reoxygenation, the ratio of the radioactivity in hypoxic cells to that in reoxygenated cells was $1.8 \pm 0.3$ (Fig. 4C). The size-exclusion HPLC analysis revealed that more than $80 \%$ of the intracellular radioactivity was derived from intact ${ }^{125}$ I-IPOS, and that approximately $70 \%$ of radioactivity in the reoxygenated medium was derived from ${ }^{125} \mathrm{I}-\mathrm{IBB}$ and other small molecules (data not shown).

\section{Biodistribution}

When we examined the biodistribution of ${ }^{125}$ I-IBB alone, the tumor-to-blood ratio was always less than 1 at each time point, indicating no tumor accumulation of ${ }^{125} \mathrm{I}-\mathrm{IBB}$ (data not shown). Next, we performed a biodistribution study of ${ }^{125} \mathrm{I}-$ IPOS. A high level of radioactivity accumulated in the liver and the spleen, and a moderate level was present in the intestine and the kidneys. Though the radioactivity in the blood at $1 \mathrm{~h}$ after injection was higher than the corresponding data for ${ }^{125} \mathrm{I}-\mathrm{IBB}(20)$, blood clearance was rapid. In contrast, the radioactivity tended to be retained in the tumor $(1.47$ $\% \mathrm{ID} / \mathrm{g}$ at $1 \mathrm{~h}$ and $1.49 \% \mathrm{ID} / \mathrm{g}$ at $6 \mathrm{~h}$ ). The tumor-to-blood ratio was greater than 1 at $24 \mathrm{~h}$ or more after injection (Table 1). We then evaluated whether the accumulation of ${ }^{125}$ I-IPOS in the tumor was dose-dependent. There were no differences in the tumor accumulation and the tumor-to-blood ratio between the 0.05- and the 0.5- $\mu$ g injected groups. However, a dose-dependent increase was observed when more than $0.5 \mu \mathrm{g}$ of ${ }^{125} \mathrm{I}$-IPOS was injected. The tumor accumulation and tumor-to-normal tissue ratio were highest in the $30-\mu \mathrm{g}$ injected group (Table 2). The tumor accumulation was 1.4 $\% \mathrm{ID} / \mathrm{g}$, the tumor-to-blood ratio was 5.1 , and the tumor-tomuscle ratio was 14.0 at $24 \mathrm{~h}$ after injection (Table 2). Evaluation $48 \mathrm{~h}$ after injection demonstrated further increases in the tumor-to-blood ratio and the tumor-to-muscle ratio (12.8 and 22.3, respectively; data not shown).

\section{In Vivo Imaging}

The tumor was clearly visualized both at 24 and at $48 \mathrm{~h}$ after ${ }^{123}$ I-IPOS injection (Fig. 5). At 24 and $48 \mathrm{~h}$ after 


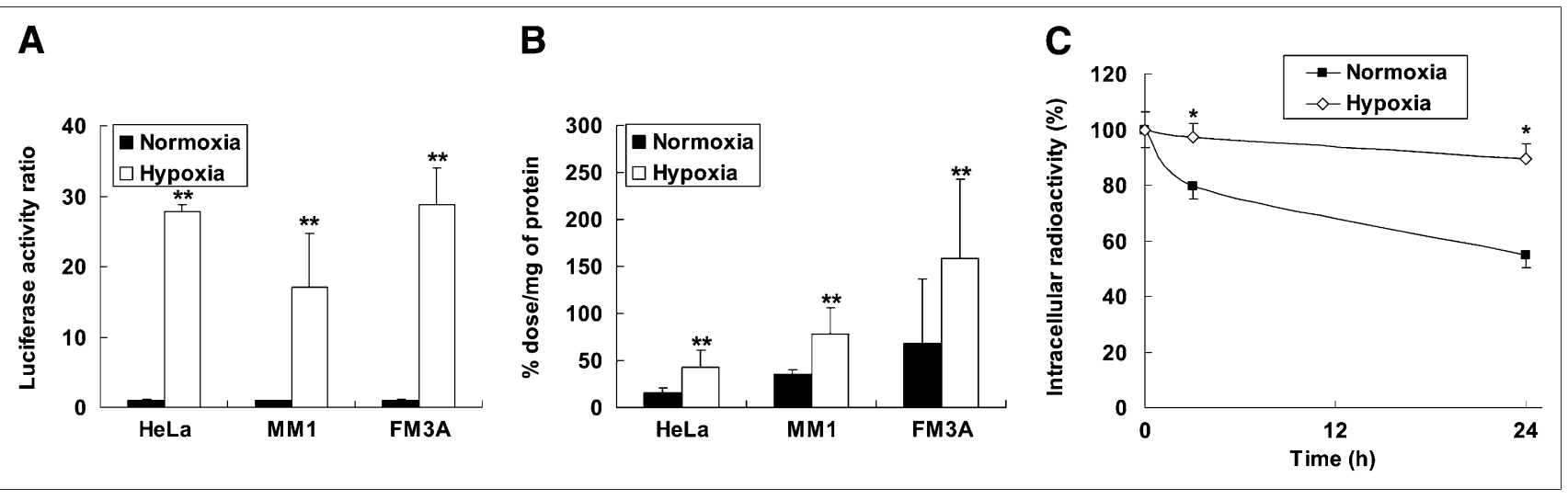

FIGURE 4. (A) HIF-1-dependent luciferase activity in HeLa, MM1, and FM3A cells. Data were normalized by protein concentration of cells. Results are shown as ratio of activity after hypoxia to activity after normoxia. Columns and bars represent mean and SD $(n=$ 3-6 ${ }^{\star \star} P<0.01$ vs. normoxia). (B) Intracellular radioactivity in HeLa, MM1, and FM3A cells after 24-h incubation under either normoxic or hypoxic conditions. Radioactivity was normalized by protein concentration of cells. Columns and bars represent mean and SD $(n=$ 3-6 ${ }^{\star \star} P<0.01$ vs. normoxia). (C) Degradation of intracellularly accumulated IPOS after reoxygenation. HeLa cells were incubated with ${ }^{125}$ I-IPOS for $24 \mathrm{~h}$ under hypoxic conditions. Then, medium was replaced with fresh medium, and cells were subjected to further incubation under normoxic or hypoxic conditions. Results are shown as percentage of radioactivity at start of second incubation. Symbols and bars represent mean and SD $\left(n=3-5 ;{ }^{\star} P<0.05\right.$ vs. normoxia).

injection, the calculated tumor-to-muscle ratios were $9.6 \pm$ 4.7 and $20 \pm 21$, respectively $(n=6)$. Although the accumulation of ${ }^{123}$ I-IPOS in the abdominal region was high, there was little accumulation in the thoracic region.

\section{Comparison Between Tumoral Accumulation of 125I-IPOS and HIF-1 Activity In Vivo}

Figure 6 shows the highly significant correlation between luciferase activity and radioactivity $(R=0.71, P<0.05)$.

\section{Regional Distribution of ${ }^{125}$ |-IPOS Relative to Hypoxic Regions in Tumor}

Pimonidazole immunohistochemistry demonstrated hypoxic areas within the tumor (Fig. 7B). On the other hand, the section derived from pimonidazole-untreated mice was not stained (Fig. 7D). The autoradiogram represents the distribution of ${ }^{125}$ I-IPOS in the tumor and shows that the distri- bution is heterogeneous and for the most part corresponds to the pimonidazole-positive hypoxic areas. However, there were also a few pimonidazole-positive regions that did not show accumulation of ${ }^{125}$ I-IPOS (Figs. 7A and 7C).

\section{DISCUSSION}

HIF-1, which is activated by hypoxia, is associated with an aggressive cancer phenotype. Tumors expressing HIF-1 are resistant to radiation therapy and chemotherapy and as a result carry a poor prognosis. Noninvasive imaging of HIF1 activity is of great interest because it may be used to predict prognosis. In our present study, we found that POS entered into tumor cells and was degraded in an oxygen-dependent manner, ${ }^{123 / 125}$ I-IPOS accumulated in tumors and the tumors were clearly visualized by in vivo imaging, the tumoral accumulation of ${ }^{125}$ I-IPOS correlated with tumoral HIF-1

\section{TABLE 1. Biodistribution of ${ }^{125}$ I-IPOS in FM3A-Implanted Mice}

\begin{tabular}{|c|c|c|c|c|}
\hline \multirow[b]{2}{*}{ Organ } & \multicolumn{4}{|c|}{ Time after injection (h) } \\
\hline & 1 & 6 & 24 & 48 \\
\hline Blood & $15.55 \pm 1.06$ & $3.14 \pm 0.54$ & $0.18 \pm 0.12$ & $0.01 \pm 0.01$ \\
\hline Liver & $32.97 \pm 1.03$ & $13.80 \pm 0.87$ & $0.97 \pm 0.44$ & $0.30 \pm 0.06$ \\
\hline Spleen & $17.29 \pm 2.35$ & $6.07 \pm 0.73$ & $1.21 \pm 0.53$ & $0.19 \pm 0.05$ \\
\hline Kidney & $5.51 \pm 0.36$ & $2.79 \pm 0.47$ & $0.74 \pm 0.60$ & $0.09 \pm 0.02$ \\
\hline Stomach & $2.25 \pm 0.57$ & $0.62 \pm 0.32$ & $0.49 \pm 0.31$ & $0.04 \pm 0.02$ \\
\hline Neck & $2.39 \pm 0.06$ & $0.80 \pm 0.08$ & $0.09 \pm 0.04$ & $0.00 \pm 0.01$ \\
\hline Intestine & $7.96 \pm 0.09$ & $9.82 \pm 1.61$ & $0.91 \pm 0.13$ & $0.24 \pm 0.08$ \\
\hline Tumor & $1.47 \pm 1.02$ & $1.49 \pm 0.38$ & $0.28 \pm 0.08$ & $0.07 \pm 0.05$ \\
\hline Muscle & $0.94 \pm 0.65$ & $0.28 \pm 0.05$ & $0.07 \pm 0.06$ & $0.00 \pm 0.01$ \\
\hline Tumor/blood & $0.10 \pm 0.07$ & $0.49 \pm 0.18$ & $1.79 \pm 0.59$ & $6.72 \pm 6.61$ \\
\hline
\end{tabular}


TABLE 2. Effect of POS Concentration on Biodistribution of ${ }^{125}$-IPOS in FM3A-Implanted Mice at 24 Hours

After Injection

\begin{tabular}{|c|c|c|c|c|}
\hline \multirow[b]{2}{*}{ Organ } & \multicolumn{4}{|c|}{ Protein concentration $(\mu \mathrm{g})$} \\
\hline & 0.05 & 0.5 & 5 & 30 \\
\hline Blood & $0.12 \pm 0.04$ & $0.16 \pm 0.10$ & $0.16 \pm 0.01$ & $0.27 \pm 0.07$ \\
\hline Liver & $1.92 \pm 0.24$ & $1.27 \pm 0.53$ & $5.53 \pm 1.05$ & $13.61 \pm 1.90$ \\
\hline Spleen & $1.07 \pm 0.30$ & $1.15 \pm 0.41$ & $2.33 \pm 0.25$ & $7.87 \pm 2.20$ \\
\hline Kidney & $0.35 \pm 0.20$ & $0.59 \pm 0.51$ & $1.03 \pm 0.10$ & $4.32 \pm 1.87$ \\
\hline Stomach & $0.28 \pm 0.14$ & $0.55 \pm 0.42$ & $0.52 \pm 0.20$ & $0.86 \pm 0.27$ \\
\hline Neck & $0.05 \pm 0.04$ & $0.09 \pm 0.04$ & $0.11 \pm 0.02$ & $0.23 \pm 0.08$ \\
\hline Intestine & $4.57 \pm 0.82$ & $1.95 \pm 2.03$ & $2.17 \pm 0.24$ & $2.18 \pm 0.28$ \\
\hline Tumor & $0.23 \pm 0.08$ & $0.25 \pm 0.07$ & $0.49 \pm 0.09$ & $1.37 \pm 0.33$ \\
\hline Muscle & $0.05 \pm 0.03$ & $0.05 \pm 0.05$ & $0.07 \pm 0.01$ & $0.10 \pm 0.03$ \\
\hline Tumor/blood & $2.04 \pm 0.61$ & $1.61 \pm 0.10$ & $3.00 \pm 0.68$ & $5.14 \pm 0.34$ \\
\hline
\end{tabular}

activity, and the intratumoral distribution of ${ }^{125}$ I-IPOS corresponded to the hypoxic regions. These findings demonstrate the feasibility of ${ }^{123}$ I-IPOS as an imaging probe for HIF-1-active, hypoxic tumors. ${ }^{123}$ I-IPOS is the first nuclear medical imaging probe for the detection of HIF-1 activity in vivo.

Other hypoxia imaging probes such as ${ }^{18} \mathrm{~F}$-fluoromisonidazole $\left({ }^{18} \mathrm{~F}\right.$-FMISO) and ${ }^{64} \mathrm{Cu}$-diacetyl-bis(N4-methylthiosemicarbazone) $\quad\left({ }^{64} \mathrm{Cu}\right.$-ATSM) have been reported previously. The retention mechanism of both of these probes in the hypoxic regions is based on reduction reactions and the formation of covalent bonds to intracellular macromolecules (22). ${ }^{18}$ F-FMISO uptake is increased only when oxygen levels fall below $2-3 \mathrm{~mm} \mathrm{Hg}$ (23). The uptake of ${ }^{64} \mathrm{Cu}$-ATSM is also markedly increased in a sigmoidal fashion under hypoxic and anoxic conditions $(<3.8 \mathrm{~mm} \mathrm{Hg})(24,25)$. Previous immunohistochemical study showed that HIF-1 $\alpha$ is more frequently present adjacent to blood vessels than in the pimonidazole-positive regions (26), and the $\mathrm{pO}_{2}$ in the HIF1 -active regions is approximately $10-15 \mathrm{~mm} \mathrm{Hg}$ (27). Thus, neither ${ }^{18} \mathrm{~F}$-FMISO nor ${ }^{64} \mathrm{Cu}$-ATSM is suitable for completely monitoring HIF-1 activity. Recently it was reported that the expression level of HIF-1 correlates with a poor prognosis in many tumors $(8,9)$. Thus, imaging HIF-1-active regions in a tumor using ${ }^{123}$ I-IPOS has the potential to provide insight into those aspects of tumor biology most relevant to treatment design.

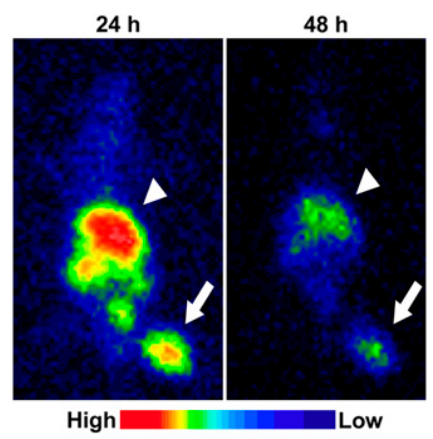

FIGURE 5. Typical planar images of FM3A-implamted mice at 24 or $48 \mathrm{~h}$ after injection of 123 I-IPOS. Tumors were clearly visualized in both images (arrow). Arrowheads indicate liver.
Protein transduction with the PTD has proven to be an effective way of delivering proteins in vitro, and recently several reports have also shown valuable in vivo applications for protein transduction in correcting disease states (28). A positive charge is important for the cellular membrane permeability of PTD. Moreover, lysine residues are required for efficient ubiquitination and after degradation of HIF- $1 \alpha$ (29). Therefore, we combined a poly-lysine sequence with a hydrophobic polypeptide to construct a unique PTD, PTD3. We characterized PTD3 in a prior study (19).

Because of its extremely high affinity and in vivo stability, we used the SAV-biotin system for radiolabeling the protein. Because the binding of ${ }^{125} \mathrm{I}-\mathrm{IBB}$ to POS was inhibited in a concentration-dependent manner by D-biotin (Fig. 2B), it was evident that ${ }^{125}$ I-IBB bound to the SAV moiety of POS, as was expected. Biotinylated probes have been used for other imaging modalities including fluorescent dyes (30) and gadolinium dendrimers (31). Thus, POS would have applications not only in nuclear medical imaging but also in optical imaging and MRI.

The luciferase activity in the hypoxic conditions was significantly higher than that in normoxic conditions (Fig. 4A), which indicates that the experimental settings used here to induce hypoxia are suitable for HIF- $1 \alpha$ stabilization. After incubation of the cells with ${ }^{125}$ I-IPOS under normoxic or hypoxic conditions, higher radioactivity was retained in the cells incubated under hypoxic conditions (Fig. 4B), and

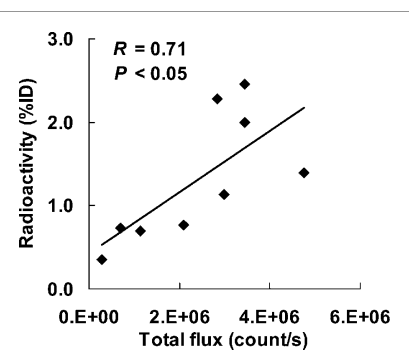

FIGURE 6. Correlation between accumulation of ${ }^{125}$ IPOS and HIF-1 activity within same tumor. Ordinate represents accumulated radioactivity (\%ID), and abscissa represents HIF-1-dependent luciferase activity. Correlation coefficient $(R)$ was 0.71 , indicating highly significant correlation $(P<0.05)$. 
A

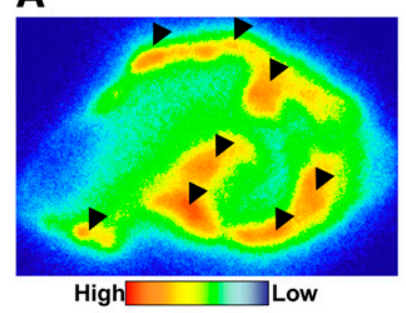

C

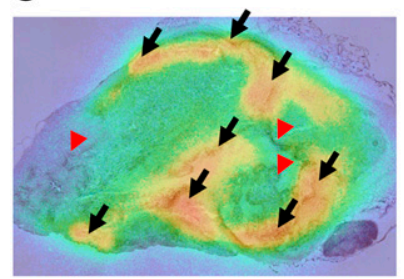

B

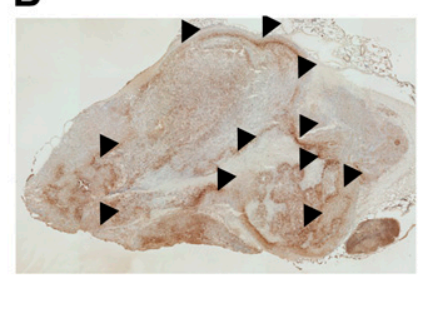

D

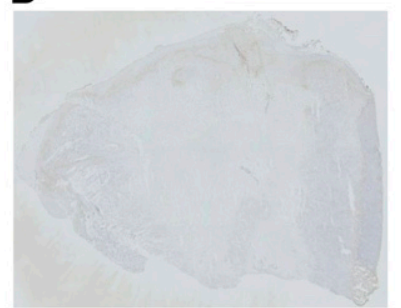

FIGURE 7. Comparison between intratumoral distribution of ${ }^{125}$ I-IPOS and pimonidazole-positive hypoxic region. Typical autoradiogram (A) and pimonidazole immunohistochemical staining (B) in identical section are shown. Merged image $(C)$ is also presented. Black arrowheads indicate area of ${ }^{125}$ I-IPOS accumulation in A and pimonidazole-positive area in B. Black arrows show areas in which both signals are positive, and red arrowheads indicate pimonidazole-positive areas without accumulation of ${ }^{125}$ I-IPOS. No signal was observed in pimonidazole-untreated tumor (D).

intracellular radioactivity under normoxic conditions decreased more rapidly than that under hypoxic conditions (Fig. 4C). The size-exclusion HPLC analysis revealed that the intracellular radioactivity was mostly derived from intact ${ }^{125}$ I-IPOS and that the radioactivity in the reoxygenated medium was derived from ${ }^{125}$ I-IBB and other small molecules. Thus, the difference in intracellular radioactivity under normoxic and hypoxic conditions probably reflects the oxygendependent degradation of POS, followed by clearance of ${ }^{125} \mathrm{I}-$ IBB from the normoxic cells. Because ${ }^{125} \mathrm{I}-\mathrm{IBB}$ is a small molecule and has adequate lipophilicity for membrane transportation, it could be cleared rapidly. However, appreciable radioactivity remained after $24-\mathrm{h}$ incubation under normoxic conditions (Figs. 4B and 4C). Though POS contains the essential domain related to the oxygen-dependent degradation of HIF-1 $\alpha$, it contains PTD and SAV as well. These modifications may lower the rate of degradation.

After injection of ${ }^{125}$ I-IPOS, high levels of radioactivity were detected in the liver, intestine, and kidneys. Because other constructs incorporating the PTD-ODD fusion proteins or SAV separately did not accumulate in the liver, the fusion of PTD-ODD with SAV may be responsible for hepatic accumulation. Multiple factors, such as molecular size, electrical charge, and slow blood clearance, may be involved. The accumulation in the kidneys may reflect either the propensity of SAV to localize in the kidneys (32) or the high HIF-1 activity in the kidneys (33). Radioactivity did not accumulate

in either the stomach or the thyroid, which demonstrates the resistance of ${ }^{125}$ I-IPOS to in vivo deiodination. Tumor accumulation of ${ }^{125}$ I-IPOS increased in a concentrationdependent manner (Table 2). As the administered concentration increased, oxygen-dependent degradation mechanisms in the normal tissues were overwhelmed, thereby increasing the quantity of POS delivered to the tumor.

In vivo imaging revealed that the tumor accumulation of ${ }^{123}$ I-IPOS correlated with HIF-1 activity. The background radioactivity in the thoracic region was quite low. Thus, ${ }^{123}$ I-IPOS would be particularly useful for detecting HIF-1 activity in lung and breast tumors. The expression of HIF-1 correlates with a poor prognosis in breast tumors (34); therefore, ${ }^{123}$ I-IPOS may be useful for the noninvasive determination of prognosis.

In the autoradiographic study, there were a few pimonidazole-positive regions in which ${ }^{125}$ I-IPOS did not accumulate. Regions in which there is no accumulation could be explained by a difference in molecular size. The significantly smaller size of pimonidazole may enable its more efficient delivery to hypoxic tissues than the larger POS. Another possible explanation is the time lag between probe injections. In the present study, ${ }^{125}$ I-IPOS was injected $22 \mathrm{~h}$ before the injection of pimonidazole. The microenvironment in tumors changes dynamically. If the oxygen level of a normoxic region decreased after the degradation of ${ }^{125} \mathrm{I}$ IPOS, pimonidazole would be able to accumulate in the region. These explanations also indicate the limitations of ${ }^{123}$ I-IPOS. The slow kinetics due to its molecular size and the necessity of prolonged time for degradation will prevent imaging of acute hypoxic regions with ${ }^{123}$ I-IPOS. However, it should be emphasized that ${ }^{125}$ I-IPOS mainly accumulated both in the pimonidazole-positive regions and in the regions surrounding them. This result is consistent with the previous results that HIF-1 expresses at higher levels of oxygen than those that allow pimonidazole metabolism and binding (26). Thus, the area in which ${ }^{125}$ I-IPOS accumulates possibly corresponds to the HIF-1-active hypoxic region.

We confirmed the specific localization of the PTD-ODD fusion protein to HIF-1-active cells using optical imaging techniques $(7,12)$. We recently constructed a novel optical imaging probe with the PTD-ODD fusion protein. We then successfully used this probe to obtain specific images of HIF-1-active cells in the ischemic brain of the focal stroke mouse model and of subcutaneous tumors in nude mice. These results further strengthen the argument that PTDODD fusion proteins including IPOS are efficiently delivered to hypoxic regions and stabilized in HIF-1-active cells.

\section{CONCLUSION}

We have developed a POS fusion protein containing the ODD domain of human HIF- $1 \alpha$. POS was degraded in an oxygen-dependent manner and was stabilized in the cells in which HIF-1 was active. Furthermore, ${ }^{123}$ I-IPOS enabled the clear visualization of the tumor in vivo and its accumulation 
correlated with HIF-1 activity in these tumors. These results support a possible role for ${ }^{123}$ I-IPOS in the imaging of HIF-1active tumor hypoxia.

\section{ACKNOWLEDGMENTS}

We thank Nihon Medi-Physics for providing the ammonium ${ }^{123}$ I-iodide. We are grateful to Hiroaki Konishi for skilled technical assistance. This study was supported in part by Health Labour Sciences Research Grant for Research on Advanced Medical Technology, from The Ministry of Health, Labour and Welfare of Japan, and a Grant-in-Aid for Exploratory Research (17659010) from The Ministry of Education, Culture, Sports, Science and Technology of Japan.

\section{REFERENCES}

1. Vaupel P, Kallinowski F, Okunieff P. Blood-flow, oxygen and nutrient supply, and metabolic microenvironment of human-tumors: a review. Cancer Res. 1989;49:6449-6465.

2. Brown JM. Exploiting the hypoxic cancer cell: mechanisms and therapeutic strategies. Mol Med Today. 2000;6:157-162.

3. Hockel M, Vaupel P. Tumor hypoxia: definitions and current clinical, biologic, and molecular aspects. J Natl Cancer Inst. 2001;93:266-276.

4. Teicher BA. Hypoxia and drug resistance. Cancer Metastasis Rev. 1994;13:139168.

5. Semenza GL. Expression of hypoxia-inducible factor 1: mechanisms and consequences. Biochem Pharmacol. 2000;59:47-53.

6. Wenger RH. Cellular adaptation to hypoxia: $\mathrm{O}_{2}$-sensing protein hydroxylases, hypoxia-inducible transcription factors, and $\mathrm{O}_{2}$-regulated gene expression. FASEB J. 2002;16:1151-1162.

7. Harada H, Kizaka-Kondoh S, Li G, et al. Significance of HIF-1-active cells in angiogenesis and radioresistance. Oncogene. 2007;26:7508-7516.

8. Marignol L, Coffey M, Lawler M, Hollywood D. Hypoxia in prostate cancer: a powerful shield against tumour destruction? Cancer Treat Rev. 2008;34:313327.

9. Miyake K, Yoshizumi T, Imura S, et al. Expression of hypoxia-inducible factor$1 \alpha$, histone deacetylase 1 , and metastasis-associated protein 1 in pancreatic carcinoma: correlation with poor prognosis with possible regulation. Pancreas. 2008;36:e1-e9.

10. Bruick RK, McKnight SL. A conserved family of prolyl-4-hydroxylases that modify HIF. Science. 2001;294:1337-1340.

11. Wang GL, Jiang BH, Rue EA, Semenza GL. Hypoxia-inducible factor 1 is a basic-helix-loop-helix-PAS heterodimer regulated by cellular $\mathrm{O}_{2}$ tension. Proc Natl Acad Sci USA. 1995;92:5510-5514.

12. Harada H, Kizaka-Kondoh S, Hiraoka M. Optical imaging of tumor hypoxia and evaluation of efficacy of a hypoxia-targeting drug in living animals. Mol Imaging. 2005;4:182-193.

13. Kizaka-Kondoh S, Inoue M, Harada H, Hiraoka M. Tumor hypoxia: a target for selective cancer therapy. Cancer Sci. 2003;94:1021-1028.

14. Harada H, Hiraoka M, Kizaka-Kondoh S. Antitumor effect of TAT-oxygendependent degradation-caspase-3 fusion protein specifically stabilized and activated in hypoxic tumor cells. Cancer Res. 2002;62:2013-2018.
15. Kageyama Y, Sugiyama H, Ayame H, et al. Suppression of VEGF transcription in renal cell carcinoma cells by pyrrole-imidazole hairpin polyamides targeting the hypoxia responsive element. Acta Oncol. 2006;45:317-324.

16. Inoue M, Mukai M, Hamanaka Y, Tatsuta M, Hiraoka M, Kizaka-Kondoh S. Targeting hypoxic cancer cells with a protein prodrug is effective in experimental malignant ascites. Int J Oncol. 2004;25:713-720.

17. Schwarze SR, Ho A, Vocero-Akbani A, Dowdy SF. In vivo protein transduction: delivery of a biologically active protein into the mouse. Science. 1999;285:1569-1572.

18. Harada H, Kizaka-Kondoh S, Hiraoka M. Mechanism of hypoxia-specific cytotoxicity of procaspase-3 fused with a VHL-mediated protein destruction motif of HIF-1 $\alpha$ containing Pro564. FEBS Lett. 2006;580:5718-5722.

19. Kizaka-Kondoh S, Itasaka S, Zeng L, et al. Selective killing of hypoxia-inducible factor-1-active cells improves survival in a mouse model of invasive and metastatic pancreatic cancer. Clin Cancer Res. In press.

20. Foulon CF, Alston KL, Zalutsky MR. Synthesis and preliminary biological evaluation of (3-iodobenzoyl)norbiotinamide and ((5-iodo-3-pyridinyl)carbonyl)norbiotinamide: two radioiodinated biotin conjugates with improved stability. Bioconjug Chem. 1997;8:179-186.

21. Ishino S, Kuge Y, Takai N, et al. ${ }^{99 \mathrm{~m} T c-a n n e x i n ~ A 5 ~ f o r ~ n o n i n v a s i v e ~ c h a r-~}$ acterization of atherosclerotic lesions: imaging and histological studies in myocardial infarction-prone Watanabe heritable hyperlipidemic rabbits. Eur J Nucl Med Mol Imaging. 2007;34:889-899.

22. Krohn KA, Link JM, Mason RP. Molecular imaging of hypoxia. J Nucl Med. 2008;49(suppl 2):129S-148S.

23. Rasey JS, Nelson NJ, Chin L, Evans ML, Grunbaum Z. Characteristics of the binding of labeled fluoromisonidazole in cells in vitro. Radiat Res. 1990; 122:301-308.

24. Lewis JS, McCarthy DW, McCarthy TJ, Fujibayashi Y, Welch MJ. Evaluation of ${ }^{64} \mathrm{Cu}$-ATSM in vitro and in vivo in a hypoxic tumor model. J Nucl Med. 1999;40:177-183.

25. Vavere AL, Lewis JS. Cu-ATSM: a radiopharmaceutical for the PET imaging of hypoxia. Dalton Trans. 2007;4893-4902.

26. Sobhanifar S, Aquino-Parsons C, Stanbridge EJ, Olive P. Reduced expression of hypoxia-inducible factor- $1 \alpha$ in perinecrotic regions of solid tumors. Cancer Res. 2005;65:7259-7266.

27. Jiang BH, Semenza GL, Bauer C, Marti HH. Hypoxia-inducible factor 1 levels vary exponentially over a physiologically relevant range of $\mathrm{O}_{2}$ tension. Am J Physiol. 1996;271:C1172-C1180.

28. Chauhan A, Tikoo A, Kapur AK, Singh M. The taming of the cell penetrating domain of the HIV Tat: myths and realities. J Control Release. 2007;117:148-162.

29. Paltoglou S, Roberts BJ. HIF- $1 \alpha$ and EPAS ubiquitination mediated by the VHL tumour suppressor involves flexibility in the ubiquitination mechanism, similar to other RING E3 ligases. Oncogene. 2007;26:604-609.

30. Ebner A, Marek M, Kaiser K, et al. Application of biotin-4-fluorescein in homogeneous fluorescence assays for avidin, streptavidin, and biotin or biotin derivatives. Methods Mol Biol. 2008;418:73-88.

31. Zhu W, Okollie B, Bhujwalla ZM, Artemov D. PAMAM dendrimer-based contrast agents for MR imaging of Her-2/neu receptors by a three-step pretargeting approach. Magn Reson Med. 2008;59:679-685.

32. Forster GJ, Santos EB, Smith-Jones PM, Zanzonico P, Larson SM. Pretargeted radioimmunotherapy with a single-chain antibody/streptavidin construct and radiolabeled DOTA-biotin: strategies for reduction of the renal dose. J Nucl Med. 2006;47:140-149.

33. Safran M, Kim WY, O'Connell F, et al. Mouse model for noninvasive imaging of HIF prolyl hydroxylase activity: assessment of an oral agent that stimulates erythropoietin production. Proc Natl Acad Sci USA. 2006;103:105-110.

34. Trastour C, Benizri E, Ettore F, et al. HIF-1 $\alpha$ and CA IX staining in invasive breast carcinomas: prognosis and treatment outcome. Int J Cancer. 2007; 120:1451-1458. 